\title{
A rare case of pancreatic adenocarcinoma and subsequent metastatic insulinoma causing severe hypoglycemia
}

\author{
Josephine H. Li*1, Catherine J. Tang ${ }^{2}$, James V. Hennessey ${ }^{2}$ \\ ${ }^{1}$ Department of Medicine, Beth Israel Deaconess Medical Center, Boston, MA, USA \\ ${ }^{2}$ Division of Endocrinology, Department of Medicine, Beth Israel Deaconess Medical Center, Boston, MA, USA
}

Received: June 19, 2016

DOI: $10.5430 /$ crim.v3n4p22
Accepted: August 21, 2016

Online Published: August 30, 2016

\begin{abstract}
Objective: To report an unusual case of concurrent pancreatic adenocarcinoma and metastatic insulinoma causing severe hypoglycemia in a patient with diabetes.

Methods: The clinical presentation, biochemical studies, and relevant imaging of this patient are presented and the pertinent literature is reviewed.

Results: A 59-year-old man with a history of unresectable pancreatic adenocarcinoma and diabetes was admitted for persistent hypoglycemia, meeting all criteria for Whipple's triad and requiring high-dose dextrose infusion. Prior outpatient abdominal imaging had revealed hepatic lesions that were biopsied and found to be a high-grade neuroendocrine carcinoma. On admission, laboratory evaluation revealed inappropriately suppressed beta-hydroxybutyrate levels, and inappropriately elevated insulin, C-peptide, and proinsulin levels. An octreotide scan revealed uptake in the hepatic lesions but not in the pancreatic head mass. Immunohistochemistry staining of prior liver biopsy samples was negative for insulin. Diazoxide therapy was initiated, but discontinued after the onset of hypotension. The hypoglycemia resolved only after trans-arterial chemoembolization of lesions both in the left and the right liver lobes, in combination with continued octreotide. Unfortunately, he subsequently developed recurrent hypoglycemia three weeks later and ultimately passed away after transitioning to hospice care.

Conclusions: Insulinomas are rare neoplasms of the pancreas that are characterized by insulin hypersecretion, which can be debilitating and potentially life-threatening. The diagnosis of metastatic insulinoma is difficult to make in the setting of multiple comorbidities and requires precise biochemical studies. Multiple medical treatments are available but the overall prognosis is poor.
\end{abstract}

Key Words: Pancreatic adenocarcinoma, Metastatic insulinoma, Hypoglycemia, Neuroendocrine carcinoma

\section{INTRODUCTION}

Insulinomas are rare neuroendocrine tumors that develop from pancreatic beta cells, and less than $10 \%$ of cases are malignant. ${ }^{[1]}$ The diagnosis of insulinoma is usually based on symptoms of hypoglycemia and biochemical studies that support inappropriate insulin hypersecretion. Malignant disease is defined as the presence of metastases, most commonly to the lymph nodes or the liver. ${ }^{[2]}$ The clinical presentation of malignant insulinoma is similar to that of more benign forms, but may be more severe and prolonged due to a higher

\footnotetext{
${ }^{*}$ Correspondence: Josephine H. Li, MD; Email: jhli@bidmc.harvard.edu; Address: Beth Israel Deaconess Medical Center, Harvard Medical School, 330 Brookline Ave, Boston, MA 02215, USA.
} 
degree of insulin overproduction. ${ }^{[3]}$ This case illustrates the occurrence of metastatic insulinoma causing severe hypoglycemia in a patient with known pancreatic adenocarcinoma and diabetes.

\section{Case presentation}

A 59-year-old male presented to the emergency department with diaphoresis and altered mental status. His initial glucose fingerstick was $20 \mathrm{mg} / \mathrm{dl}$. Correction of his hypoglycemia alleviated his symptoms, consistent with the presence of Whipple's triad. Administration of multiple ampules of 50\% dextrose produced an initial rise in glucose but his serum glucose subsequently continued to drop. Due to persistent hypoglycemia, the patient was admitted to the intensive care unit on continuous $10 \%$ dextrose.

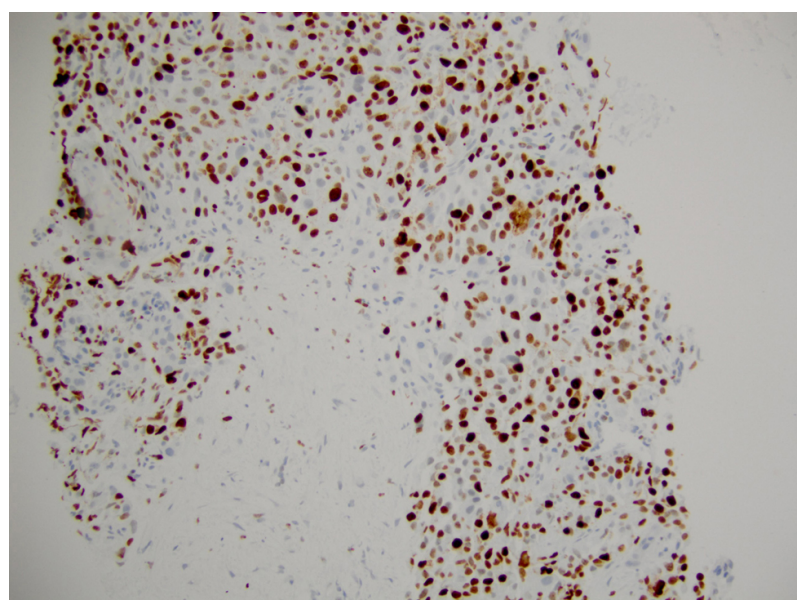

Figure 1. Immunohistochemical staining for MIB-1 of high-grade neuroendocrine tumor with proliferation index of approximately 80\% (Images courtesy of Robert Najarian, MD)

His past medical history was notable for pancreatic adenocarcinoma, diagnosed 2.5 years earlier biochemically and by endoscopic ultrasound-guided fine needle aspiration. He had undergone neoadjuvant chemotherapy with FOLFIRINOX and had been taken for attempted surgical resection, but his tumor was deemed unresectable due to lymph node metastases. He had subsequently received stereotactic radiotherapy and several cycles of gemcitabine and nab-paclitaxel. Unfortunately, a CT scan three months prior to admission had identified liver lesions concerning for metastases. A liver biopsy had unexpectedly revealed a WHO/ENETs Grade 3 well-differentiated neuroendocrine carcinoma with a proliferation fraction of approximately $80 \%$ on MIB- 1 immunostaining (see Figure 1), and the patient had undergone further palliative chemotherapy with carboplatin and etoposide. A subsequent CT scan performed just prior to his admission had revealed interval growth of the liver lesions, with the

Published by Sciedu Press largest measuring $4 \mathrm{~cm} \times 5.9 \mathrm{~cm}$ in the left lobe. The known pancreatic head mass measured $2 \mathrm{~cm} \times 2.7 \mathrm{~cm}$, and there was a $5.3 \mathrm{~cm} \times 7.4 \mathrm{~cm}$ mesenteric lymph node conglomerate anterior to the pancreas (see Figure 2).

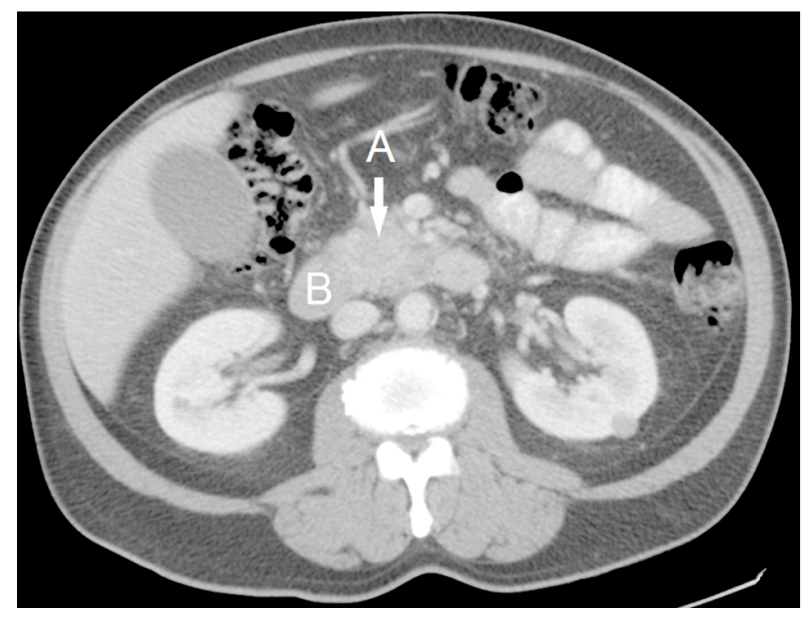

Figure 2. $\mathrm{CT}$ abdomen with and without contrast at initial diagnosis. (A) Hypodense ill-defined mass in the head of the pancreas; (B) Duodenum (Images courtesy of J. Anthony Parker, MD)

Apart from his oncologic history, the patient also had a history of hypertension and type 2 diabetes. His diabetes was diagnosed at the same time he was found to have the pancreatic mass, and he had been initiated on metformin with initial improvement in his hemoglobin A1c. He later required both long-acting and rapid-acting insulins for glycemic control. However, his insulin and metformin had been discontinued one month prior to admission due to hypoglycemia. Table 1 provides a detailed timeline of the patient's oncologic history and treatment of diabetes from the time of diagnosis.

On admission to the intensive care unit, the patient was afebrile with tachycardia to 102 beats per minute. Physical examination was otherwise unremarkable. A venous glucose drawn while symptomatic was $32 \mathrm{mg} / \mathrm{dl}$, and the patient simultaneously had an elevated C-peptide $5.29 \mathrm{ng} / \mathrm{ml}(0.8$ $3.85 \mathrm{ng} / \mathrm{ml})$, elevated insulin $67.1 \mu \mathrm{IU} / \mathrm{ml}(2-19.6 \mu \mathrm{IU} / \mathrm{ml})$, elevated proinsulin $182.4 \mathrm{pmol} / \mathrm{L}(\leq 18.8 \mathrm{pmol} / \mathrm{L})$, and low beta-hydroxybutyrate $0.1 \mathrm{mmol} / \mathrm{L}(<0.4 \mathrm{mmol} / \mathrm{L})$. A sulfonylurea screen was negative and insulin auto-antibodies were undetectable. Adrenal insufficiency was ruled out with a cortisol response to $38.8 \mathrm{mcg} / \mathrm{dl}$ at 60 minutes after administration of $250 \mathrm{mcg}$ of cosyntropin. Because of the high suspicion for insulinoma, the patient's prior liver biopsy was sent for insulin staining of the original pathology blocks but returned negative. 
As a result of the increasing dextrose requirements, the patient was started on diazoxide $75 \mathrm{mg}$ every 8 hours orally. This was increased to $150 \mathrm{mg}$ every 8 hours, but the patient developed worsening hyponatremia and hypotension. Due to his clinical deterioration, the diazoxide was discontinued. The patient underwent an octreotide scan, which revealed uptake in the liver but interestingly not within the pancreatic head mass related to his known adenocarcinoma (see Figure 3). After octreotide scanning, he was treated with octreotide $50 \mathrm{mcg}$ twice daily, subcutaneously, in conjunction with up to $100 \mathrm{cc} /$ hour of $50 \%$ dextrose.
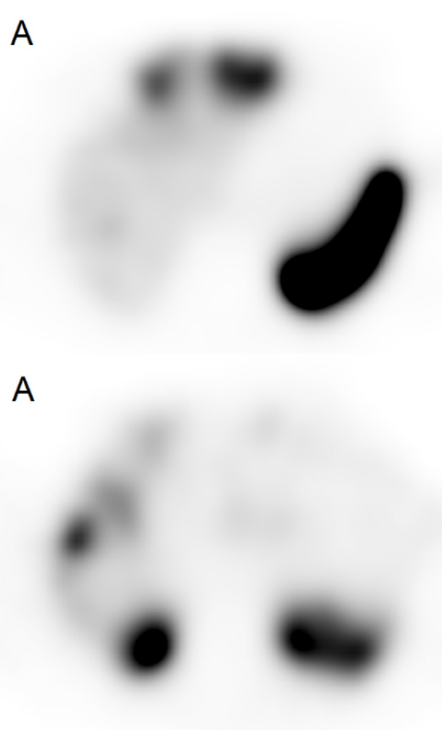

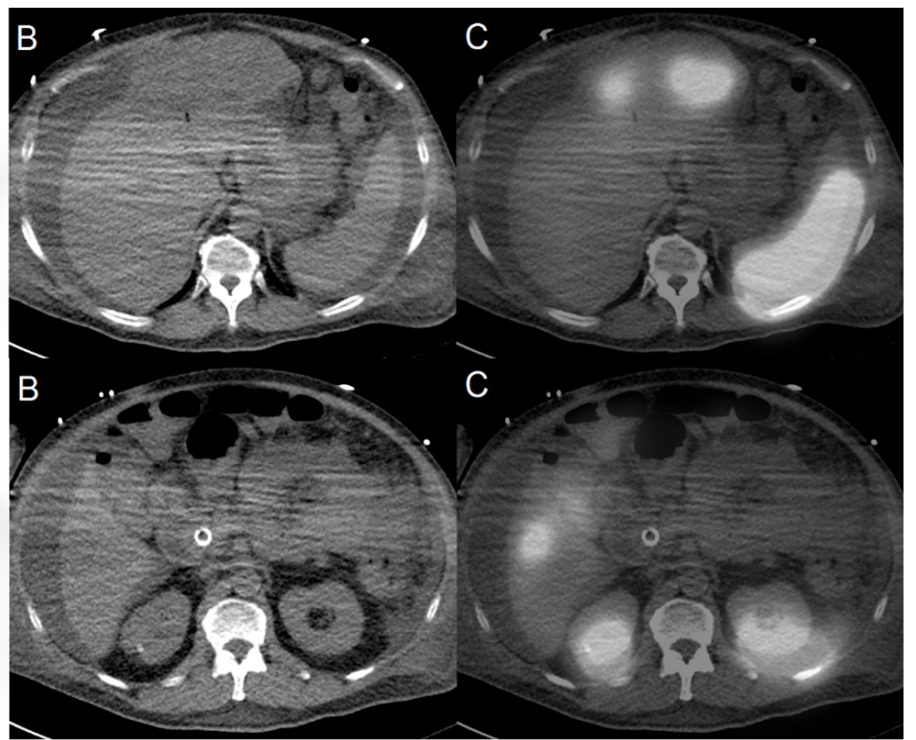

Figure 3. Top panel (A) Octreotide scan showing multiple foci of abnormal pentetreotide uptake corresponding to (B) hepatic lesions seen on computed tomography scan. (C) Overlay of A on B. The spleen shows physiologic uptake of pentetreotide. Bottom panel (A) Octreotide scan showing multiple foci of abnormal pentetreotide uptake in the liver but no uptake in the pancreatic head mass seen on (B) computed tomography scan. (C) Overlap of A on B. The kidneys show physiologic uptake of pentetreotide. The hyperdense ring seen in the pancreas is a common bile duct stent (Images courtesy of J. Anthony Parker, MD)

Trans-arterial chemoembolization (TACE) of the metastatic neuroendocrine tumor was performed, first to the left liver lobe, followed by the right liver lobe. The patient quickly became normoglycemic on octreotide $100 \mathrm{mcg}$ twice daily monotherapy and he was weaned off octreotide a week after the second TACE. Unfortunately, his hospital course was further complicated by healthcare-associated pneumonia, multidrug-resistant bacteremia, and recurrent ascites. In another two weeks, he again developed hypoglycemia and was transitioned to comfort care. He was discharged to hospice and passed away a few days later.

\section{DiscuSSION}

Insulinoma has an incidence of only 4 cases per million person per year, of which fewer than $10 \%$ of cases are malignant. $^{[1]}$ In contrast, almost 50,000 people in the United States each year are diagnosed with cancer of the exocrine pancreas, and $85 \%$ of these are adenocarcinomas. ${ }^{[4]}$ As such, the coexistence of both pancreatic adenocarcinoma and insulinoma is exceedingly rare, and to our knowledge, only one previous case has been reported in the literature. In this one case, an individual was discovered to have a $1.5 \mathrm{~cm}$ solid pancreatic mass that was resected and found to contain a $1 \mathrm{~mm}$ pancreatic adenocarcinoma on histopathology. However, successive abdominal CT scans later additionally revealed a $2 \mathrm{~cm}$ pancreatic mass that was successfully treated surgically with a Whipple's procedure. Histopathology of this second lesion unexpectedly revealed an insulinoma. ${ }^{[5]}$ To our knowledge, our case is the first case of concurrent pancreatic adenocarcinoma and metastatic insulinoma. Moreover, while most insulinomas reported in the literature are of WHO/ENETs Grade 1 or 2, corresponding to proliferation indices of $1 \%-20 \%$, our case describes an insulinoma with a remarkably high proliferation rate of $80 \%$.

There were several interesting features of this case. At the time of the patient's diagnosis of pancreatic adenocarcinoma, he was also found to have diabetes. This highlights the link between the two diseases, where recently diagnosed type 2 diabetes of two years or less has been associated with a greater risk of pancreatic cancer. ${ }^{[6]}$ The mechanism is not 
entirely clear, but is believed to be due to induced insulin resistance rather than gland destruction by tumor. Patients who undergo pancreatic tumor resection experience improvement or resolution of diabetes despite removal of variable amounts of pancreas, supporting the hypothesis that the tumor itself induces diabetes. ${ }^{[7,8]}$ While there are no established screening guidelines for pancreatic cancer, new-onset type 2 diabetes in an older individual with weight loss might be an early manifestation of pancreatic cancer. ${ }^{[9]}$ In this particular case, the patient's history of diabetes possibly delayed the diagnosis of malignant insulinoma since he was on hypoglycemic agents, as has been observed by previous authors. ${ }^{[10]}$

The patient had a biopsy-proven neuroendocrine tumor in his liver and biochemical studies consistent with an insulinoma. The best explanation of these findings is that he developed a separate metastatic insulinoma in addition to his pancreatic adenocarcinoma, as evidenced by the octreotide scan showing increased uptake only in the liver lesions but not at the known pancreatic head mass. Moreover, the patient's prior liver biopsy did not stain positive for insulin, though the tissue was from two months prior to his hospitalization, before the onset of his severe hypoglycemia. This finding further raised our suspicion that the insulinoma was not present at the time of his pancreatic adenocarcinoma diagnosis, and that the new liver lesions represented a new, distinct, neoplastic process. There are a few caveats, of note, to this conclusion. Not all insulinomas express sufficient somatostatin receptors to allow for visualization on octreotide scanning, and thus the lack of uptake in the pancreas may be due to the poor sensitivity of the octreotide scan. ${ }^{11]}$ In fact, the sensitivity of octreotide scanning for the detection of insulinomas has been reported to be approximately $50 \% .{ }^{[11]}$ Thus, it is possible that the insulinoma arose from the pancreatic head but without enough somatostatin receptors to be visualized. Alternatively, octreotide uptake from a pancreatic tail mass could have been mistaken for physiologic uptake by the left kidney, as has been reported previously. ${ }^{[11]}$ And though immunohistochemical confirmation that his original pancreatic biopsy specimen was negative for neuroendocrine markers would have further supported our hypothesis that the insulinoma was a de novo neoplasm, unfortunately there was inadequate tissue remaining for staining. Prior to the discovery of his insulinoma, the patient also received standard chemotherapy agents for his pancreatic adenocarcinoma, including FOLFIRINOX (which is comprised of folinic acid, fluorouracil, irinotecan, and oxaliplatin), gemcitabine, and nab-paclitaxel (see Table 1). In our literature search, we did not find evidence of an association between any of these agents and possible differentiation of the pancreatic adenocarcinoma to insulinoma.

Table 1. Timeline of the patient's oncologic history and management of his type 2 diabetes

\begin{tabular}{|c|c|c|}
\hline Month/Year & Oncologic Event & Diabetes Management \\
\hline $1 / 2013$ & $\begin{array}{l}\text { Admitted for painless jaundice. Discovered to have pancreatic } \\
\text { head mass. }\end{array}$ & $\begin{array}{l}\text { Random glucose }>400 \text { during hospitalization and A1c } \\
9.1 \% \text {. Started on metformin } 500 \mathrm{mg} \text { BID. }\end{array}$ \\
\hline $2 / 2013-3 / 2013$ & $\begin{array}{l}\text { Pancreatic adenocarcinoma diagnosed on FNA of pancreatic head } \\
\text { mass. Started neoadjuvant chemotherapy with FOLFIRINOX. }\end{array}$ & \\
\hline $6 / 2013$ & Underwent planned Whipple but tumor deemed unresectable. & A1c of $7.5 \%$. Continued on same regimen. \\
\hline 7/2013-10/2013 & $\begin{array}{l}\text { Underwent } 1 \text { month of CyberKnife stereotactic body radiotherapy } \\
\text { and } 6 \text { cycles of FOLFIRINOX. }\end{array}$ & \\
\hline $12 / 2013-6 / 2014$ & Surveillance CT scans without evidence of disease progression. & $\begin{array}{l}\text { Metformin increased to } 750 \mathrm{mg} \text { BID. A1c of } 11.7 \% \text { in } \\
6 / 2014 \text {. }\end{array}$ \\
\hline $8 / 2014$ & & Lantus initiated and increased to 22 units daily. \\
\hline 9/2014-11/2014 & $\begin{array}{l}\text { CT scan showed local disease progression. Started chemotherapy } \\
\text { with gemcitabine. }\end{array}$ & Novolog was added to the patient's regimen. \\
\hline $2 / 2015$ & $\begin{array}{l}\text { Nab-paclitaxel added to gemcitabine due to continued disease } \\
\text { progression on CT. }\end{array}$ & $\begin{array}{l}\text { Oncology notes documented poor glycemic control due to } \\
\text { loss of insurance. }\end{array}$ \\
\hline $5 / 2015-6 / 2015$ & CT scan showed new liver metastases. & $\begin{array}{l}\text { Started to have sporadic hypoglycemic episodes in } \\
\text { mid-June. A1c of } 8.1 \% \text {. }\end{array}$ \\
\hline $7 / 2015$ & Liver biopsy revealed WHO Grade 3 neuroendocrine carcinoma. & \\
\hline $8 / 2015$ & Initiated on palliative chemotherapy with carboplatin/etoposide. & $\begin{array}{l}\text { Hypoglycemic events became more frequent to several } \\
\text { times a week. Discontinued off metformin and insulin. }\end{array}$ \\
\hline 9/2015 & $\begin{array}{l}\text { CT scan showed marked interval progression of hepatic lesions } \\
\text { and mesenteric lymph node conglomerate. }\end{array}$ & Admitted for persistent hypoglycemia. \\
\hline
\end{tabular}


Due his unresectable metastases, the patient received several treatment modalities. He was started on diazoxide, a benzothiadiazine that decreases insulin secretion and increases gluconeogenesis. ${ }^{[12]}$ While it is first-line therapy and may provide the greatest long-term benefit for insulinoma patients, diazoxide can cause adverse effects such as edema, digestive intolerance, and hirsutism. ${ }^{[2]}$ It is also an antihypertensive agent, so there is a risk of hypotension, which was the reason for its discontinuation in this case. Octreotide, a somatostatin analogue, is a second option, but the response is contingent upon the presence of somatostatin receptor-expressing insulinoma. ${ }^{[12]}$ Moreover, it inhibits glucagon secretion, which limits its ability to correct hypoglycemia. While octreotide appears to control hypoglycemia in over $50 \%$ of patients with insulinoma, it was more likely to be ineffective in patients with metastatic disease to the liver. ${ }^{[13]}$

Ultimately, the patient's hypoglycemia was reversed after undergoing TACE, which can be effective in cases of severe hypoglycemia. In one case series of 10 patients with malignant insulinoma, the median survival after TACE was 2.6 years, which is longer than the average survival of less than two years. ${ }^{[2,14]}$ However, a study of TACE for liver metastases of neuroendocrine tumors included two insulinoma patients who initially responded but had recurrent episodes of hypoglycemia at 3-6 months and 4-6 weeks, respectively. ${ }^{[15]}$ This was similarly seen in the current case, as the patient had marked initial success but later succumbed to relapsing hypoglycemia. Because the patient was critically ill with multiple infections, he was transitioned to comfort care and did not pursue further treatment.

However, there exist other potential secondary therapies for refractory hypoglycemia, which include radiofrequency ablation (RFA) and additional chemotherapy agents. RFA involves the use of high-frequency electrical waves that generate heat and result in targeted tissue destruction. While RFA has been shown to provide sustained relief of hor- monal symptoms in 89 patients with various neuroendocrine liver metastases, its long-term efficacy in the treatment of metastatic insulinoma has not been clearly established. ${ }^{[16]}$ Streptozocin-based chemotherapies traditionally have been the standard treatment for well-differentiated neuroendocrine tumors whereas high-grade and poorly-differentiated tumors appear to respond better to platinum-based chemotherapies. ${ }^{[17,18]}$ The patient likely did not receive a streptozocinbased agent due to the high-grade nature of his carcinoma and was instead initiated on palliative carboplatin and etoposide prior to his admission for hypoglycemia. Had this patient been able to undergo further treatment during his hospitalization, everolimus, an mTOR-inhibitor, may have been a potential candidate. In small case series of patients with insulinoma and refractory hypoglycemia, treatment with everolimus resulted in substantial improvement in glycemic control. ${ }^{[19,20]}$ The mechanism of action is thought to be due to a direct effect of everolimus on insulin production or release.

In summary, we report an unusual case of unresectable pancreatic adenocarcinoma with diabetes and the subsequent discovery of a metastatic insulinoma causing severe refractory hypoglycemia. Diagnosing metastatic insulinoma can be challenging in the presence of multiple comorbidities such as co-existing malignancy and diabetes requiring hypoglycemic agents. Medical therapies include diazoxide, octreotide, TACE, RFA, and chemotherapy agents, but appear to provide limited benefit in the face of an aggressive tumor.

\section{ACKNOWLEDGEMENTS}

Written informed consent was obtained from the patient's wife for publication of this report.

\section{CONFLICTS OF INTEREST DISCLOSURE}

The authors have declared no conflicts of interest.

\section{REFERENCES}

[1] Service JF, McMahon MM, O'Brien PC, et al. Functioning insulinoma-incidence, recurrence, and long-term survival of patients: a 60-year study. Mayo Clin Proc. 1991; 66: 711-719. http://dx.doi .org/10.1016/S0025-6196(12)62083-7

[2] Hirshberg B, Cochran C, Skarulis MC, et al. Malignant insulinoma Cancer. 2005; 104: 264-272. http://dx.doi.org/10.1002/cnc r. 21179

[3] Baudin E, Caron P, Lombard-Bohas C, et al. Malignant insulinoma: Recommendations for characterisation and treatment. Ann Endocrinol (Paris). 2013; 74: 523-533. http://dx.doi.org/10. $1016 / j$. ando. 2013.07.001
[4] Siegel RL, Miller KD, Jemal A. Cancer statistics, 2015. CA Cancer J Clin. 2015; 65: 5-29. http: //dx . doi .org/10.3322/caac. 21 254

[5] Athanasopoulos PG, Polymeneas G, Dellaportas D, et al. Concurrent insulinoma and pancreatic adenocarcinoma: report of a rare case and review of the literature. World J Surg Oncol. 2011; 9: 7 http://dx.doi.org/10.1186/1477-7819-9-7

[6] Gullo L, Pezzilli R, Morselli-Labate AM. Diabetes and the risk of pancreatic cancer. N Engl J Med. 1994; 331: 81-84. http: //dx.doi.org/10.1056/NEJM199407143310203

[7] Permert J, Adrian TE, Jacobsson P, et al. Is profound peripheral insulin resistance in patients with pancreatic cancer caused by a 
tumor-associated factor? Am J Surg. 1993; 165: 61-67. http: //dx.doi.org/10.1016/S0002-9610(05) 80405-2

[8] Pannala R, Leirness JB, Bamlet WR, et al. Prevalence and clinical profile of pancreatic cancer-associated diabetes mellitus. Gastroenterology. 2008; 134: 981-987. http://dx.doi.org/10.1053/j .gastro.2008.01.039

[9] Pannala R, Basu A, Petersen GM, et al. New-onset diabetes: a potential clue to the early diagnosis of pancreatic cancer. Lancet Oncol. 2009; 10: 88-95. http://dx.doi.org/10.1016/S1470-204 $5(08) 70337-1$

[10] Schmitt J, Boullu-Sanchis S, Moreau F, et al. Association of malignant insulinoma and type 2 diabetes mellitus: a case report. Ann Endocrinol (Paris). 2008; 69: 69-72. http://dx.doi .org/10.10 $16 / j$. ando. 2007.11 .002

[11] Mirallié E, Pattou F, Malvaux P, et al. Value of endoscopic ultrasonography and somatostatin receptor scintigraphy in the preoperative localization of insulinomas and gastrinomas. Gastroenterol Clin Biol. 2002; 26: 360-6.

[12] Ferrer-Garcia J, González-Cruz VI, Navas-DeSolís S, et al. Management of malignant insulinoma. Clin Transl Oncol. 2013; 15: 725-731. http://dx.doi.org/10.1007/s12094-012-0996-7

[13] Vezzosi D, Bennet A, Rochaix P, et al. Octreotide in insulinoma patients: efficacy on hypoglycemia, relationships with Octreoscan scintigraphy and immunostaining with anti-sst2A and anti-sst5 antibodies. Eur J Endocrinol. 2005; 152: 757-767. http://dx. doi.o $\mathrm{rg} / 10.1530 /$ eje.1.01901
[14] Starke A, Saddig C, Mansfeld L, et al. Malignant metastatic insulinoma-postoperative treatment and follow-up. World J Surg. 2005; 29: 789-793. http://dx.doi.org/10.1007/s00268-005 $-7743-\mathrm{y}$

[15] Kress O, Wagner HJ, Wied M, et al. Transarterial chemoembolization of advanced liver metastases of neuroendocrine tumors-a retrospective single-center analysis. Digestion. 2003; 68: 94-101. http://dx.doi.org/10.1159/000074522

[16] Akyildiz HY, Mitchell J, Milas M, et al. Laparoscopic radiofrequency thermal ablation of neuroendocrine hepatic metastases: long-term follow-up. Surgery. 2010; 148: 1288-1293. http://dx.doi.org /10.1016/j.surg. 2010.09.014

[17] Kulke MH, Anthony LB, Bushnell DL, et al. NANETS treatment guidelines: well-differentiated neuroendocrine tumors of the stomach and pancreas. Pancreas. 2010; 39: 735. http://dx.doi.org/10. 1097/MPA. 0b013e3181ebb168

[18] Sorbye H, Welin S, Langer SW, et al. Predictive and prognostic factors for treatment and survival in 305 patients with advanced gastrointestinal neuroendocrine carcinoma (WHO G3): the NORDIC NEC study. Ann Oncol. 2012: mds276. http://dx.doi.org/10. 1093/annonc/mds276

[19] Kulke MH, Bergsland EK, Yao JC. Glycemic control in patients with insulinoma treated with everolimus. N Engl J Med. 2009; 360: 195-197. http://dx.doi.org/10.1056/NEJMc0806740

[20] Bernard V, Lombard-Bohas C, Taquet MC, et al. Efficacy of everolimus in patients with metastatic insulinoma and refractory hypoglycemia. Eur J Endocrinol. 2013; 168: 665-674. http://dx .doi.org/10.1530/EJE-12-1101 